\title{
EVALUACIÓN DE LA ACTIVIDAD LEISHMANICIDA Y TOXICIDAD AGUDA DEL EXTRACTO HIDROALCOHÓLICO DE LOS TALLOS DE Croton alnifolius
}

\author{
Evaluation of leishmanicidal activity and acute toxicity of hydroalcoholic extract from stems \\ of Croton alnifolius
}

\author{
Luis A Inostroza ${ }^{1}$, Américo J Castro ${ }^{1}$, Eloisa M Hernández ${ }^{1}$, Hugo E Casanova ${ }^{2}$. \\ ${ }^{1}$ Facultad de Farmacia y Bioquímica, Universidad Nacional de San Marcos. ${ }^{2}$ Facultad de Farmacia y Bioquímica, Universidad \\ Nacional de Trujillo
}

\section{RESUMEN}

En el presente estudio se evaluaron la actividad leishmanicida y la toxicidad aguda oral del extracto hidroalcohólico de los tallos de Croton alnifolius. Para lo primero, se infectaron con promastigotes de Leishmania peruviana, en la pata trasera derecha, a Mesocricetus auratus (hámster); después de tres semanas los animales se trataron con el extracto hidroalcohólico de Croton alnifolius. El efecto del extracto fue contrastado con estibogluconato sódico a $60 \mathrm{mg}$ de Sbv/kg por día, durante 20 días. El potencial tóxico del extracto hidroalcohólico fue evaluado mediante el ensayo de toxicidad aguda oral en ratones, determinándose la dosis letal media $\left(\mathrm{DL}_{50}\right)$ con tres niveles de dosis del extracto (2000, 200 y $50 \mathrm{mg} / \mathrm{kg}$ ), evaluándose el peso corporal y el estudio histopatológico; también se evaluó su citotoxicidad contra Artemia salina. Se encontró que el extracto posee actividad leishmanicida comparable con el estibogluconato sódico. La $\mathrm{DL}_{50}$ estimada fue de 1396,114 mg/kg, clasificándose el extracto de Croton alnifolius como ligeramente tóxico; apreciándose ligeras alteraciones vasculares del tipo congestión en el examen histopatológico. En general, no se evidenció daño considerable ni modificaciones en sus órganos, y la concentración letal media sobre Artemia salina fue de 40,9 $\mu \mathrm{g} / \mathrm{mL}$ considerándose ligeramente tóxico. Fueron identificados los principales grupos de metabolitos secundarios presentes en el extracto mediante un tamizaje fitoquímico. Los resultados obtenidos permiten validar la seguridad del uso tradicional del extracto hidroalcohólico de los tallos secos de Croton alnifolius para tratar problemas de salud relacionados con leishmaniasis.

Palabras clave: Croton alnifolius, actividad leishmanicida, toxicidad aguda, dosis letal media.

\section{SUMMARY}

This study evaluated the leishmanicidal activity and acute oral toxicity of hydroalcoholic extract from stems of Croton alnifolius. For the first, were infected with promastigotes of Leishmania peruviana, in the right hind leg, Mesocricetus auratus (hamster); after three weeks the animals were treated with the hydroalcoholic extract of Croton alnifolius. The effect of the extract was compared with sodium stibogluconate at $60 \mathrm{mg} \mathrm{SbV} \mathrm{/} \mathrm{kg} \mathrm{per} \mathrm{day} \mathrm{for} 20$ days. The toxic potential of hydroalcoholic extract was evaluated using the acute oral toxicity test on mice, determining the media lethal dose $\left(\mathrm{LD}_{50}\right)$ with three dose levels of extract (2000, 200 and $50 \mathrm{mg} / \mathrm{kg}$ ), assessing body weight and histopathological study, also was evaluated their cytotoxicity against Artemia salina. It was found that the extract has leishmanicidal activity comparable to sodium stibogluconate. The $\mathrm{LD}_{50}$ value was estimated to $1396,11 \mathrm{mg} / \mathrm{kg}$, the Croton alnifolius extract was classified as slightly toxic, because caused slight alterations such vascular congestion in the histopathological examination. In general no evidence of significant damage or changes in their bodies, and the media lethal concentration of Artemia salina was 40,9 g/mL considered slightly toxic. Were identified the main groups of secondary metabolites present in the extract through a phytochemical screening. The results obtained allow to validate the safety of the traditional use of hydroalcoholic extract of the dried stems of Croton alnifolius to treat health problems related to leishmaniasis.

Keywords: Croton alnifolius, leishmanicidal activity, acute toxicity, media lethal dose.

\section{INTRODUCCIÓN}

a leishmaniasis es una enfermedad causada por protozoos del género Leishmania. La infección correspondea una antropozoonosis que llega al hombre por la picadura de insectos infectados. Aproximadamente 12 millones de personas, en 88 países, sufren de leishmaniasis; sin embargo, los enfermos se encuentran principalmente en los países en vías de desarrollo, bajo diversas formas clínicas según la especie de Leishmania responsable y los órganos afectados: visceral, mucocutánea y cutánea. Algunas de éstas evolucionan gravemente si no son tratadas, y pueden ser fatales. Es preferentemente tropical y subtropical, siendo transmitida por dípteros hematófagos del género 
Lutzomyia, en el Nuevo Mundo, y Phlebotomus, en el Viejo Mundo, a partir de reservorios constituidos por roedores y pequeños mamíferos. El parásito se encuentra en dos formas: extracelular, flagelado o promastigote libre, que vive en el tubo digestivo y la saliva del insecto, e intracelular o amastigote que se halla en células del sistema retículoendotelial y en los fagolisosomas de los macrófagos del mamífero que lo aloja ${ }^{(1)}$.

Los antimoniales pentavalentes, desarrollados en 1940, incluyendo al antimoniato de $\mathrm{N}$-metilglucamina y el estibogluconato sódico, continúan siendo considerados agentes de primera línea para el tratamiento de las leishmaniasis. Los esquemas de tratamiento son largos, costosos y la eficacia de la terapéutica farmacológica dista de ser completa, observándose resistencia del parásito y toxicidad cada vez más frecuentes. Los principales problemas en el uso de estas quimioterapias son las reacciones adversas que producen, destacándose: toxicidad cardiovascular con anomalías electrocardiográficas, bradicardia, vasodilatación, shock, trastornos renales y hepáticos. Por otro lado, se trata de medicamentos de difícil acceso por parte de las comunidades rurales ${ }^{(2)}$. Se estima que el $80 \%$ de la población mundial utiliza las plantas medicinales como la única fuente de tratamiento terapéutico frente a diversas enfermedades. A pesar del gran avance de los últimos años en la industria farmacéutica, drogas modernas no están disponibles son demasiado costosas para las personas de bajos recursos económicos. La mayoría de fármacos activos contra agentes infecciosos son derivados de recursos naturales $o$ a partir de estructuras sugeridas por éstos. Estos hechos impulsan al desarrollo de nuevos medicamentos que sean más eficaces, de menor toxicidad, bajo costo y que reemplacen o complementen a los usados actualmente en la práctica clínica antileishmaniásica.

Por lo anteriormente mencionado y debido a la ausencia de confirmación experimental del uso etnobotánico, se realizó el presente trabajo de investigación para evaluar la actividad leishmanicida del extracto hidroalcohólico de los tallos de Croton alnifolius en hámsteres dorados con leishmaniasis cutánea, y su efecto tóxico agudo oral en ratones albinos, además de un bioensayo de citotoxicidad sobre Artemia salina e identificación de los principales grupos de metabolitos secundarios; de modo tal que pueda ser una alternativa de uso leishmanicida y sin riesgo para la población.

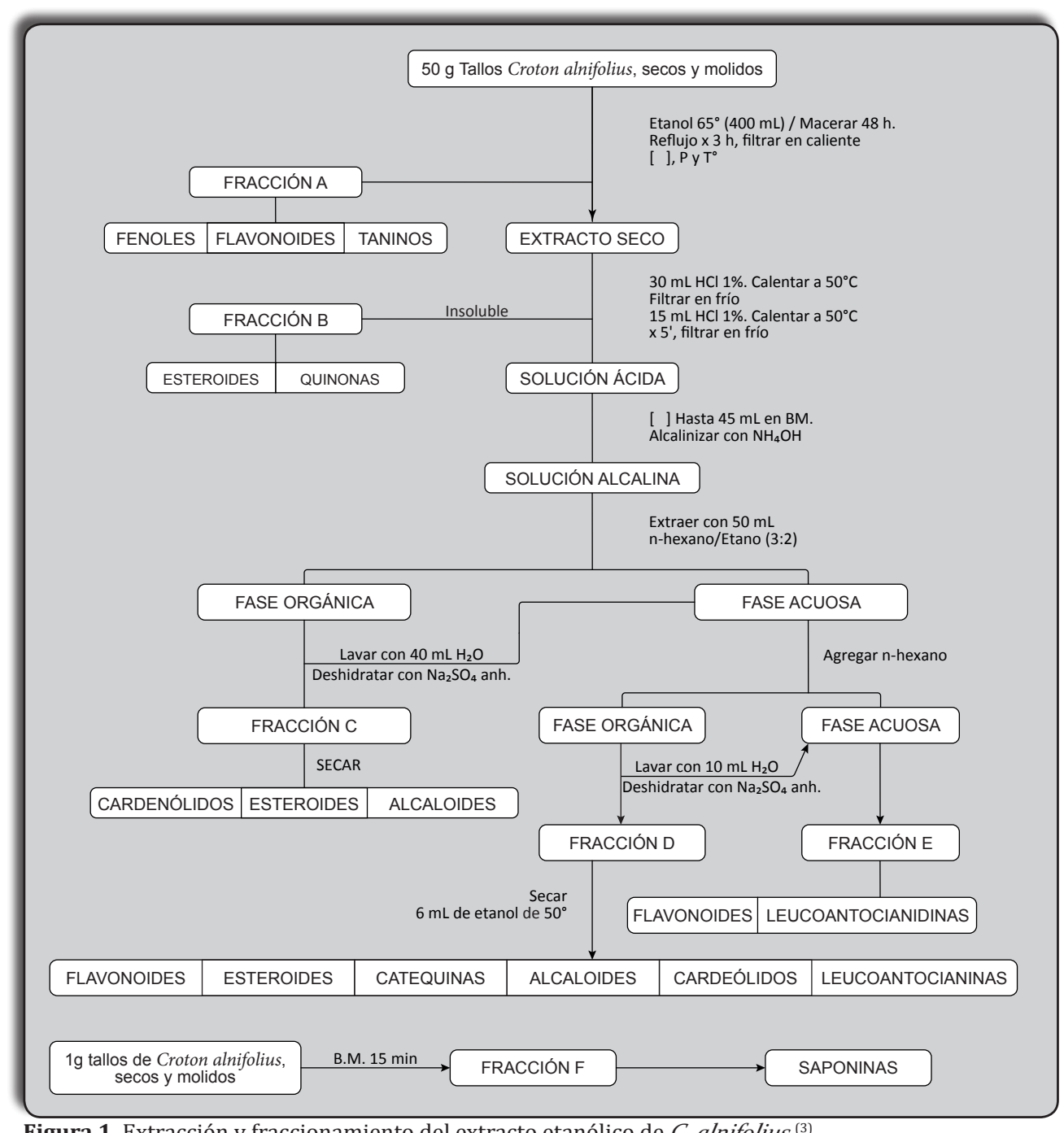

Figura 1. Extracción y fraccionamiento del extracto etanólico de C. alnifolius $^{(3)}$ 


\section{MATERIALES Y MÉTODO}

El diseño del estudio es de tipo experimental, longitudinal y prospectivo, habiéndose realizado en el laboratorio de Química Aplicada a las Ciencias de la Salud de la Facultad de Farmacia y Bioquímica de la Universidad Nacional de Trujillo (UNT) y en el laboratorio de Química Orgánica de la Facultad de Farmacia y Bioquímica de la Universidad Nacional Mayor de San Marcos (UNMSM).

\section{Material Vegetal}

Croton alnifolius "tunga", colectado en la zona reservada de Chancaybaños, ubicada entre 1300 y 2400 $\mathrm{m}$, distrito de Chancaybaños, provincia de Santa Cruz, región de Cajamarca. Los ejemplares colectados fueron confrontados con los depositados en el Herbarium Truxillensis de la Universidad Nacional de Trujillo. Luego fueron trasladados al laboratorio de Química Aplicada a la Ciencias de la Salud de la Facultad de Farmacia y Bioquímica de la UNT, donde fueron secados a la sombra, a temperatura ambiente y sobre papel, durante siete días. Terminado este proceso se procedió a la pulverización en molino de cuchillas, obteniéndose un polvo grueso de $2 \mathrm{~mm}$ que se guardó en frascos de vidrio colorámbar para su empleo en la elaboración del extracto hidroalcohólico, posterior identificación de principios bioactivos y pruebas biológicas correspondientes.

\section{Técnica de extracción de los principales metabolitos secundarios}

Para la marcha fitoquímica se procedió a obtener las diferentes fracciones, según se detalla en la figura $1^{(3)}$.

\section{Estudio fitoquímico}

Con cada una de las fracciones obtenidas se realizaron las reacciones químicas de identificación para metabolitos secundarios: flavonoides (Shinoda y amoniaco), compuestos fenólicos (cloruro férrico), alcaloides (Dragendorff, Mayer, Hager y Wagner), triterpenosyesteroides (Liebermann-Buchard), quinonas (Bortrager), compuestos lactónicos y cumarinas (Baljet), antocianidinas (Rosemhein), taninos (cloruro férrico y gelatina) y saponinas (Molisch) ${ }^{(4)}$.

\section{ENSAYOS BIOLÓGICOS}

\section{Actividad leishmanicida in vivo ${ }^{(5)}$ \\ Cultivo y mantenimiento de parásitos}

Se mantuvieron promastigotes de $L$. (V.) peruviana en medio Schereider (Sigma) con 10\% de suero fetal bovino, $10000 \mathrm{U} / \mathrm{mL}$ de penicilina, $10000 \mathrm{mg} / \mathrm{mL}$ de estreptomicina y $200 \mathrm{mM}$ de L-glutamina.

Se recolectaron los promastigotes en la fase logarítmica tardía de crecimiento por centrifugación a 3 ooo rpm, se lavaron tres veces en solución salina amortiguada con fosfatos (PBS, $\mathrm{pH} \mathrm{8,o}$ ) y, finalmente, se resuspendieron en medio fresco y se ajustaron a una concentración de $1 \times 10^{6} / \mathrm{mL}$.

Cuantificación de promastigotes para inoculación Se realizaron diluciones 1:10 para el recuento de promastigotes, separando los cúmulos de parásitos succionando repetidas veces con micropipetas; una alícuota de esta dilución se llevó inmediatamente a la cámara de Neubauer para el respectivo recuento. Se contaron los parásitos encontrados en la totalidad de los cuadrantes. Para determinar el número de parásitos presentes en cada mililitro se empleó la siguiente fórmula:

$\mathrm{N}^{\circ}$ parásitos $=\left(\mathrm{N}^{\circ}\right.$ células $)($ factor dilución $)($ factor conversión de cámara $)$

Después de conocer el número de parásitos por cada mililitro se ajustó la concentración a $1 \times 10^{6}$ parásitos $/ \mathrm{mL}$.

\section{Animales e infección experimental}

Se utilizaron para este ensayo 30 Mesocricetus auratus (hámsteres) hembras, de 55-6o g, que permanecieron en cuarentena durante 7 días antes de comenzar el estudio. Se mantuvieron en una habitación con temperatura controlada de $22 \pm 2^{\circ} \mathrm{C}$ y ciclo luz/oscuridad 12/12 horas. La alimentación consistió en dieta estándar para ratas y ratones y agua a voluntad. Se formaron tres grupos de 10 animales cada uno, un grupo experimental tratado con la sustancia de prueba (Grupo A), un grupo control positivo (Grupo B) y un grupo control negativo (Grupo C).

Todos los hámsteres dorados fueron inoculados intradérmicamente en la pata derecha del tren posterior con $1 \times 10^{6}$ promastigotes procedentes de cultivo en fase estacionaria (día 6) de Leishmania peruviana.

\section{Esquema de tratamiento y evaluación de la} eficacia clínica

Tres semanas después de la inoculación con los promastigotes de L. peruviana, los hámsteres fueron tratados por vía intramuscular durante 20 días a una dosis única diaria: Grupo A con $60 \mathrm{mg}$ de extracto hidroalcohólico de tallos de Croton alnifolius por $\mathrm{kg}$ de peso; grupo B con $60 \mathrm{mg}$ de antimonio pentavalente de estibogluconato sódico por kg peso, y grupo C solución salina tamponada estéril, siguiendo el mismo esquema.

El seguimiento de la evolución del tratamiento en los Mesocricetus auratus se hizo diariamente por medición del ancho, espesor y largo de las lesiones, utilizando 
un calibrador digital. Se compararon los resultados obtenidos en los tres grupos de estudio, analizándose las diferencias de las mediciones realizadas.

La eficacia clínica del tratamiento se expresó mediante un índice de evolución de la lesión:

$$
\mathrm{IE}=\frac{\text { Tamaño de la lesión durante tratamiento }(\mathrm{mm})}{\text { Tamaño de la lesión antes de iniciar tratamiento }(\mathrm{mm})}
$$

Los registros se efectuaron en los días o, 5, 10 y 20 de tratamiento y a los 15 días después del mismo.

\section{Toxicidad aguda oral ${ }^{(6)}$}

Se emplearon 40 ratones albinos suizos cepa Balb/c, hembras, con peso promedio de $22 \mathrm{~g}$, procedentes del bioterio del Instituto Nacional de Salud. Se confeccionaron 4 grupos de 10 animales cada uno, identificados individualmente para su dosificación de acuerdo al peso corporal, mediante un sistema de marcaje con ácido pícrico, se mantuvieron en una habitación a temperatura controlada de $20 \pm 2^{\circ} \mathrm{C}$, con un ciclo de luz/oscuridad de 12/12 h. La alimentación consistió en ratonina peletizada y agua a voluntad.

El extracto hidroalcohólico de los tallos de Croton alnifolius se administró por vía oral mediante cánula intragástrica, en dosis única directamente del frasco, previo ayuno de 4 h; se ensayaron 3 niveles de dosis: $50 \mathrm{mg} / \mathrm{kg}$ (mínima), 200 $\mathrm{mg} / \mathrm{kg}$ (media) y $2000 \mathrm{mg} / \mathrm{kg}$ (máxima), con el propósito de determinar la dosis letal media $\left(\mathrm{DL}_{50}\right)$; también se ensayó un grupo control negativo, al cual se le administró el vehículo empleado. Todos los animales fueron observados constantemente durante las primeras $24 \mathrm{~h}$, continuando diariamente durante un período de catorce días y registrando cualquier signo tóxico además de la mortalidad. Al finalizar esteperíodo seprocedióal sacrificio por tracción de la nuca seguido de necropsia con examen macroscópico de los órganos y tejidos, principalmente: corazón, riñón, hígado, pulmón, estómago y cerebro, los cuales fueron fijados en formol al 10\% durante 72 horas y posteriormente procesados rutinariamente para el examen histopatológico. El peso corporal se controló al inicio y al final del experimento, expresándose en gramos.

El valor de $\mathrm{DL}_{50}$ se estimó mediante el método estadístico de los Probits, teniendo en cuenta el valor de los sólidos totales presentes en el extracto.

\section{Citotoxicidad en Artemia salina}

La prueba citotóxica in vitro para determinar la concentración letal media $\left(\mathrm{CL}_{50}\right)$ del extracto hidroalcohólico de Croton alnifolius se realizó con nauplios de Artemia salina cultivados bajo condiciones estrictas de salinidad $(3,8 \%)$ y temperatura de 25 a $28^{\circ} \mathrm{C}$, de acuerdo al protocolo de McLaughlin ${ }^{(7)}$.

\section{RESULTADOS}

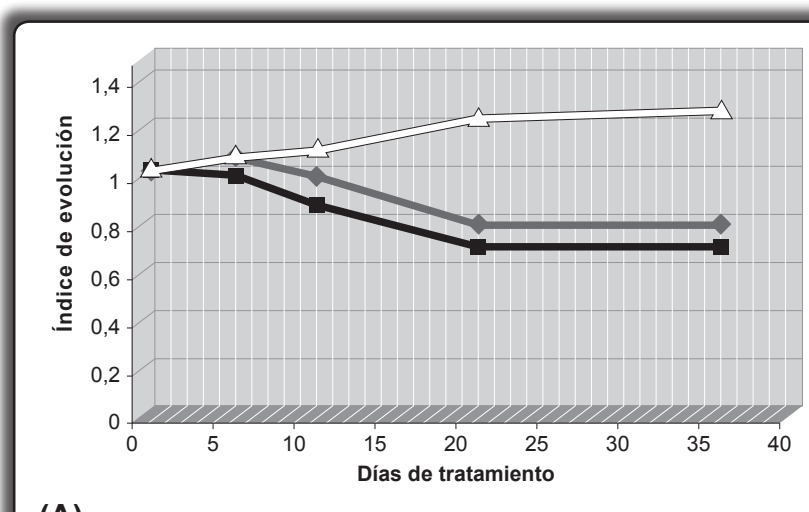

(A) $\sim$ Croton alnifolius $\rightarrow$ Estibogluconato sódico $\quad \sim$ Solución salina
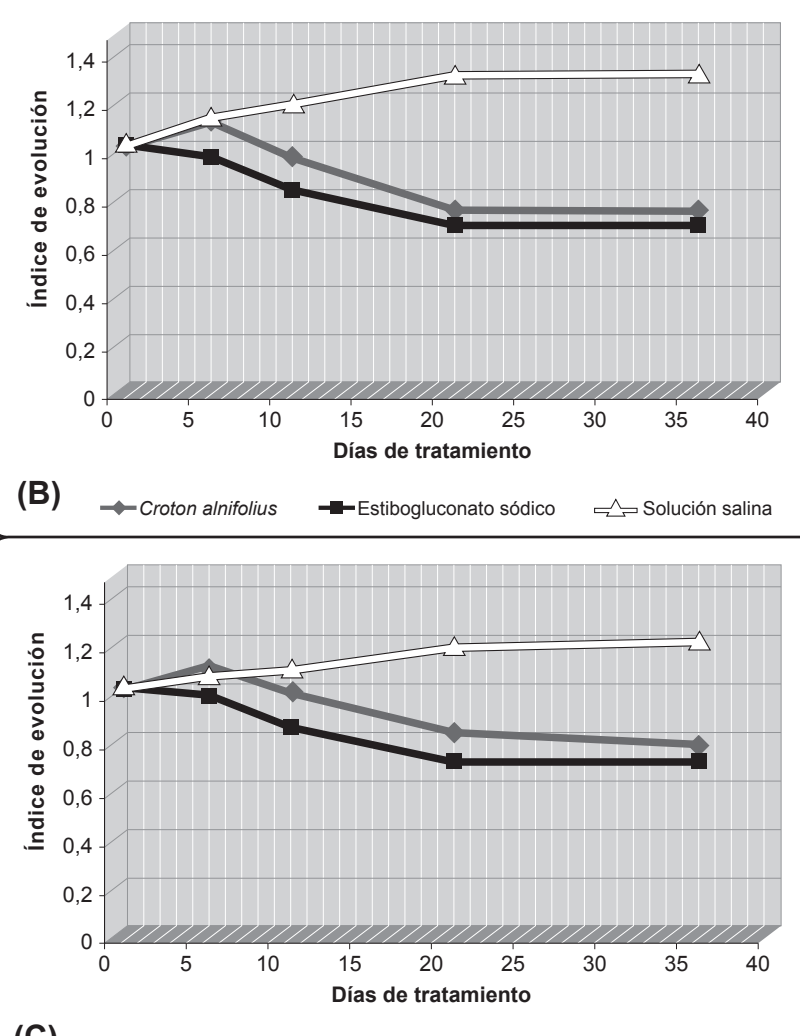

(C) $\sim$ Croton alnifolius $\quad \rightarrow$ Estibogluconato sódico $\quad \sim$ Solución salina

Figura 2. Índice de evolución de la lesión de Mesocricetus auratus infectados con promastigotes de Leishmania (V) peruviana que se trataron después de 3 semanas con extracto hidroalcohólico de tallos de Croton alnifolius (60 mg/Kg de peso, el Grupo A), estibogluconato sódico (60 mg de $\mathrm{SbV} / \mathrm{kg}$ de peso, el Grupo B) y solución salina (60 $\mathrm{mg} / \mathrm{Kg}$ de peso, el Grupo C), por día durante 20 días. (A) Índice de evolución del ancho de lesión. (B) Índice de evolución del espesor de lesión y (C) Índice de evolución del largo de lesión. El índice de evolución se calcula al dividir el tamaño de la lesión en un tiempo determinado $\left(\mathrm{T}_{\mathrm{j}}\right)$ sobre el tamaño de la lesión antes de iniciar el tratamiento $\left(\mathrm{T}_{0}\right)$ : IE $=\left(\mathrm{T}_{\mathrm{i}}\right) /\left(\mathrm{T}_{0}\right)$. 
Tabla 1. Metabolitos secundarios en 6 fracciones diferentes del extracto hidroalcohólico al 65\% de tallos de Croton alnifolius.

\begin{tabular}{|c|c|c|c|}
\hline Fracción & $\begin{array}{l}\text { Metabolitos } \\
\text { secundarios }\end{array}$ & Reacción & Resultado \\
\hline \multirow{5}{*}{ A } & Fenoles & $\mathrm{FeCl}_{3}$ & + \\
\hline & \multirow{2}{*}{ Flavonoides } & Shinoda & + \\
\hline & & Amoniaco & + \\
\hline & \multirow{2}{*}{ Taninos } & $\mathrm{FeCl}_{3}$ & + \\
\hline & & Gelatina & + \\
\hline \multirow{2}{*}{ B } & Esteroides & Liebermann-Burchard & + \\
\hline & Quinonas & Bortranger & + \\
\hline \multirow{6}{*}{$\mathrm{C}$} & Cardenólidos & Baljet & + \\
\hline & Esteroides & Liebermann-Burchard & - \\
\hline & \multirow{4}{*}{ Alcaloides } & Dragendorff & + \\
\hline & & Hager & + \\
\hline & & Mayer & + \\
\hline & & Wagner & + \\
\hline \multirow{9}{*}{$\mathrm{D}$} & \multirow{2}{*}{ Flavonoides } & Shinoda & - \\
\hline & & Amoniaco & - \\
\hline & \multirow[t]{2}{*}{ Esteroides } & Liebermann-Burchard & - \\
\hline & & Dragendorff & - \\
\hline & \multirow{3}{*}{ Alcaloides } & Hager & - \\
\hline & & Mayer & - \\
\hline & & Wagner & - \\
\hline & Cardenólidos & Baljet & - \\
\hline & Leucoantocianidinas & Rosemhein & - \\
\hline \multirow{3}{*}{$\mathrm{E}$} & Leucoantocianidinas & Rosemhein & - \\
\hline & \multirow{2}{*}{ Flavonoides } & Shinoda & + \\
\hline & & Amoniaco & + \\
\hline \multirow{2}{*}{$\mathrm{F}$} & \multirow{2}{*}{ Saponinas } & Espuma & + \\
\hline & & Molisch & + \\
\hline
\end{tabular}

(+) Significa una respuesta positiva para este metabolito

(-) Significa una respuesta negativa para este metabolito

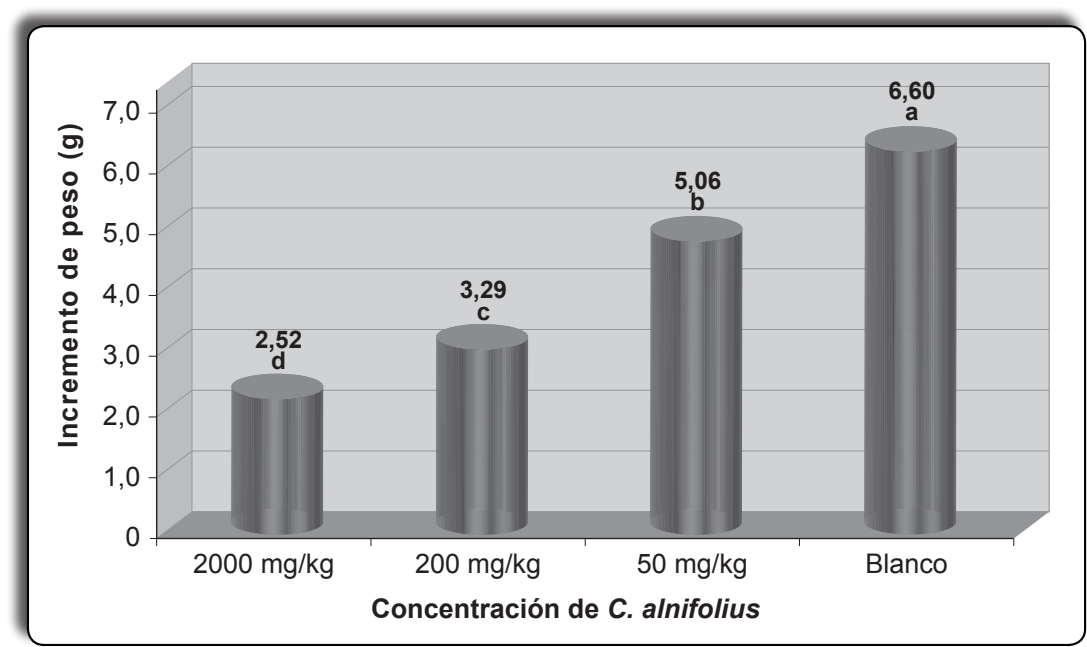

Figura 3. Prueba de Tukey de la toxicidad del extracto hidroalcohólico de los tallos de Croton alnifolius expresado como variación del incremento de peso en ratones albinos.

\section{DISCUSIÓN}

La leishmaniasis es tratada comúnmente con antimonio pentavalente en la forma de estibogluconato
Tabla 2. Dosis administrada, signos/síntomas y dosis letal media del extracto hidroalcohólico de los tallos de Croton alnifolius en ratones albinos.

\begin{tabular}{clc}
\hline $\begin{array}{c}\text { Dosis } \\
\text { administradas } \\
\text { (mg/kg) }\end{array}$ & \multicolumn{1}{c}{ Signos/Síntomas } & $\begin{array}{c}\text { Dosis letal } \\
\text { media } \\
\text { (mg/kg) }\end{array}$ \\
\hline 2000 & $\begin{array}{l}\text { Depresión profunda, incoordina- } \\
\text { ción motora, respiración acelerada, } \\
\text { piloerección, arrastra el tren pos- }\end{array}$ & \\
& $\begin{array}{l}\text { terior, sedación, reestablecimiento } \\
\text { Se registraron muertes de un animal } \\
\text { en el noveno día y dos animales en }\end{array}$ & \\
& $\begin{array}{l}\text { el onceavo día del período de ob- } \\
\text { servación en el grupo que recibió } \\
\text { tratamiento con la dosis máxima }\end{array}$ & $1,396,11$ \\
& $\begin{array}{l}\text { (2000mg/kg). } \\
200\end{array}$ & $\begin{array}{l}\text { No se registraron muertes en los gru- } \\
\text { pos a los que se les administró las do- }\end{array}$ \\
\hline 50 & sis 200 y 50 mg/Kg. \\
\hline
\end{tabular}

Tabla 3. Valores de $\mathrm{CL}_{50}$ del extracto hidroalcohólico de los tallos de Croton alnifolius y del control, sulfato de vinblastina, empleando el bioensayo con Artemia salina.

\begin{tabular}{cc}
\hline Sustancia & $\mathbf{C L}_{50}(\boldsymbol{\mu g} / \mathbf{m L})$ \\
\hline Extracto hidroalcohólico de C. alnifolius & 40,9 \\
Sulfato de vinblastina & 13,45 \\
\hline
\end{tabular}

de sodio o antimoniato de $\mathrm{N}$-metilglucamina y con pentamidina o anfotericina $\mathrm{B}$, drogas potencialmente tóxicas y generalmente administradas en hospitales. En las regiones endémicas, los tratamientos son llevados a cabo empleando la medicina popular aprovechando las propiedades de las biomóleculas presentes en los vegetales disponibles en la zona ${ }^{(8)}$.

Se obtuvieron respuestas positivas para una gran diversidad de grupos funcionales de compuestos, entre los que se destacaron: alcaloides, flavonoides, quinonas, fenoles, taninos, cardenólidos, esteroides y saponinas (Tabla 1). Los resultados obtenidos del tamizaje fitoquímico fueron similares a los logrados por Casanova et al ${ }^{(3)}$.

Seconfirmólapresenciadealcaloides que son el principio activo al cual se le atribuye la propiedad leishmanicida de esta planta, dicha actividad en los mecionados alcaloides ha sido demostrada por varios investigadores. Tempone et al ${ }^{(9)}$ reportaron la actividad in vitro de alcaloides isoquinolínicos, contenidos en extractos de Annona coriacea y Annona crassiflora, entre otras plantas, contra promastigotes de Leishmania chagasi. Otros metabolitos secundarios también ejercen actividad contra Leishmania; así por ejemplo, las saponinas triterpenoides obtenidas de 


\section{Careya arborea atacan a Leishmania donovani ${ }^{(10)}$.}

Aunque no se han encontrado referencias respecto al efecto leishmanicida de Croton alnifolius, similares a lo que afirman Alzamora et al ${ }^{(11)}$ sobre Lepidium peruvianum; la presencia en esta en esta última especiede alcaloides imidazólicos, benzilglucosinolatos, taninos, flavonoides y saponinas, todos ellos compuestos muy activos frente a Leishmania brazilensis y Leishmania peruviana, serían los causantes del efecto leishmanicida.

En el presente estudio, para evaluar la actividad leishmanicida se empleó un modelo animal (hámster) en conjunto con una cepa e inóculo definido de Leishmania, lo cual eliminó algunos factores de confusión como: 1) la respuesta inmune variable del hospedero, 2) el tiempo postinfección, 3) el sitio de la lesión y la heterogeneidad en la infectividad/ patogenicidad de Leishmania ${ }^{(12)}$.

Según el seguimiento clínico de los hámsteres tratados con estibogluconato sódico, medicamento de referencia; extracto hidroalcohólico de tallos de $C$. alnifolius; y un grupo control negativo, que no recibió tratamiento leishmanicida luego de ser infectados experimentalmente con promastigotes de Leishmania peruviana; se encontró, al finalizar el tratamiento que tanto el estibogluconato sódico como el extracto hidroalcohólico de Croton alnifolius mostraron diferencias clínicas significativas con respecto al grupo no tratado (figura 2).

Los ensayos in vivo, como en el presente estudio, son esenciales para la evaluación de los antimoniales y posiblemente son aplicables a otros compuestos, debido a que los resultados obtenidos in vitro carecen del componente farmacocinético y farmacodinámico, y los vehículos como el m-clorocresol (Estibogluconato sódico) y bisulfito de potasio y sulfito de sodio anhidro (Glucantime $^{\circledR}$ ) pueden modificar el efecto antiLeishmania ${ }^{(5)}$.

Aponte et al (13) evidenciaron el efecto farmacológico del $C$. alnifolius estudiando su actividad citotóxica, debido a un metabolito identificado como 12-O-tetradecanoilforbol-13-acetato. Además mostró actividad in vitro contra cepas de Mycobacterium tuberculosis.

El presente estudio tiene correlación con los resultados de Estévez et al ${ }^{(14)}$, quienes afirman que el C. alnifolius y otras especies vegetales son utilizadas en la medicina popular amazónica por diferentes grupos étnicos para resolver problemas de leishmaniasis y demostraron que sus extractos etanólicos poseen actividad leishmanicida in vitro frente a Leishmania amazonensis.

Además, el género Croton contiene alcaloides, como: taspina, flavonoides y aceites esenciales; estos últimos pueden contener compuestos destacados como anetol y linalol. Posiblemente dichos metabolitos estén actuando en sinergismo contra la leishmaniasis (Leishmania amazonensis) ${ }^{(15)}$.

De acuerdo a la dosis letal media del extracto hidroalcohólico de los tallos de Croton alnifolius $\left(\mathrm{DL}_{50}=1396,11 \mathrm{mg} / \mathrm{kg}\right)($ Tabla 2$)$, se clasifica como ligeramente tóxico, extrapolando en la tabla de Williams (menor a $5000 \mathrm{mg} / \mathrm{kg}$ ) ${ }^{(7)}$. En la necropsia realizada, no se encontraron alteraciones patológicas al análisis macroscópico de los órganos. En el análisis histopatológico se apreciaron ligeras alteraciones vasculares del tipo congestión, pero, en general, no se evidenciaron daños considerables ni modificaciones en sus órganos (pulmón, corazón, hígado y riñón).

Los pesos corporales de los ratones albinos presentaron unavariación deacuerdoa la concentración del extracto administrado según se detalla en la figura 3. Esto podría darnos a entender que los componentes del extracto hidroalcohólico del C. alnifolius, como los taninos, forman en el intestino complejos con las enzimas digestivas disminuyendo la absorción de nutrientes, especialmente azúcares y lípidos, además de provocar la excreción de altas cantidades de proteínas dietéticas y endógenas, por lo cual son considerados antinutricionales. Otros metabolitos inhiben la digestión, al afectar la actividad catalítica de algunas enzimas y pueden restringir la absorción de los alimentos. Algunos metabolitos secundarios también se han calificado de factores antinutricionales debido a que pueden causar un efecto negativo en el valor nutricional del alimento, así como en la salud animal y humana ${ }^{(16)}$. Para comprender mejor estos procesos es necesaria la realización de nuevos estudios.

Al estudiar los metabolitos bioactivos de una planta, debe identificarse preliminarmente el grado de actividad biológica, para ello el método de nauplios de Artemia salina es un bioensayo versátil y estandarizado que permite monitorear la presencia de compuestos citotóxicos y antitumorales de utilidad potencial. Un extracto o sustancia es considerada potencialmente útil como citotóxico y antitumoral cuando su CL5o es $\leq 30$ $\mu \mathrm{g} / \mathrm{mL}{ }^{(7)}$. La concentración letal media del extracto hidroalcohólico de $C$. alnifolius) fue de $40,9 \mu \mathrm{g} / \mathrm{mL}$ (Tabla 3). Este valor que es superior al 13,45 $\mu \mathrm{g} / \mathrm{mL}$ correspondiente al el sulfato de vinblastina, lo presenta como un posible agente citotóxico y antitumoral, talvés 
debidoa la acción individual deuno de sus constituyentes, o a un sinergismo entre algunos de ellos.

\section{CONCLUSIONES}

El extracto hidroalcohólico de los tallos de Croton alnifolius presenta similar actividad leishmanicida que estibogluconato sódico frente a Leishmania peruviana inducida en Mesocricetus auratus.

La $\mathrm{DL}_{50}$ del extracto fue de 1396,11 $\mathrm{mg} / \mathrm{kg}$ clasificándose como ligeramente tóxico de acuerdo a la tabla de Williams (menor a $5000 \mathrm{mg} / \mathrm{kg}$ ). En el examen histopatológico se apreciaron ligeras alteraciones vasculares del tipo congestión. En general no se evidenció daño considerable ni modificaciones en sus órganos (pulmón, corazón, hígado y riñón).

La concentración de inhibición media sobre Artemia salina fue de $40,9 \mu \mathrm{g} / \mathrm{mL}$ presentando el extracto de $C$. alnifolius ligera citotoxicidad.

El tamizaje fitoquímico del extracto hidroalcohólico de Croton alnifolius indica la presencia de abundante cantidad de alcaloides, flavonoides, quinonas, fenoles, taninos, cardenólidos, esteroides y saponinas; que actuando sinérgicamente serían los responsables de la actividad leishmanicida.

\section{REFERENCIAS BIBLIOGRÁFICAS}

1. Botero D, Restrepo M. Parasitosis Humanas. $4^{\mathrm{a}}$ ed. Corporación para Investigaciones Biológicas. Medellín, 2003. p. 238-6o.

2. Flórez J. Farmacología Humana. $5^{\text {a }}$ ed. Elsevier-Masson. Barcelona, 2008. p. 1361-90.

3. Casanova H. Separación de metabolitos secundarios de plantas medicinales e identificación de metabolitos secundarios con reactivos de coloración y precipitación. $\mathbf{1}^{\mathrm{a}}$ ed. Ed. La Libertad. Trujillo, 2006.

4. Victoria MC, Morón F, Morejón Z, Martínez MJ, López M. Tamizaje fitoquímico, actividad antiinflamatoria y toxicidad aguda de extractos de hojas de Annona squamosa L. Rev Cubana Plant Med 2006; 11(1). [En línea] Acceso 02 Mayo 2009. Disponible en: http:// scielo.sld.cu/scielo.php?script=sci_arttext\&pid=S102847962006ooo100oo2\&lng=es

5. Henao HH, Osorio Y, Saravia NG, Gómez A, Travi BL. Eficacia y toxicidad de los antimoniales pentavalentes (Glucantime $^{\circledR}$ y Pentostam ${ }^{\circledR}$ ) en un modelo animal de leishmaniasis cutánea americana: aplicación de la luminometría. Biomédica 2004; 24(4): 393-402.
6. Martínez MJ, Betancour J, Ramírez AR, Barceló H, Meneses R, Lainez A. Evaluación toxicológica aguda de los extractos fluidos al 30 y 80\% de Cymbopogon citratus (D.C.) Stapf (caña santa). Rev Cubana Plant Med 200o; 5(3): 97-101.

7. Meyer BN, Ferrigni NR, Putnam JE, Jacobsen LB, Nichols DE, McLaughlin JL. Brine shrimp: a convenirnt general bioassay for active plant constituyents. Planta Med 1982; 45: 31-4.

8. Hernández JE, Tenorio JL, Rojas CM. Evaluation of the leishmanicidal activity of the ethanolic extracts of Rollinia rufinervis on Leishmania chagasi. Vitae 2005; 12 (2): 37-43.

9. Tempone AG, Treiger SE, Andrade HF, Amorin NC, Yogi A, Salerno C et al. Antiprotozoal activity of Brazilian plants extract from isoquinoline alkaloid-producing families. Phytomedicine 2005; 12: 382-90.

10. Manda D, Panda N, Kumar S, Banerjee S, Mandal N, Sahu N. A triterpenoid saponin possessing antileishmanial activity from the leaves of Careya arborea. Phytochemistry 2006; 67(2): 183-90.

11. Echevarría A, Torres D. Efecto de un extracto de Petiveria alliacea Lin sobre el crecimiento de Giardia lamblia in vitro. Rev Cubana Med Milit 2001; 30: 161-5.

12. Sinagra A, Riarte A, Luna C, Campanini A, Segura EL. Leishmania (Viannia) braziliensis: biological behavior in golden hamsters of isolates from Argentine patients. Am J Trop Med Hyg 1997; 57: 115-8.

13. Aponte J, Vaisberg A, Rojas R, Caviedes L, Lewis W, Lamas $\mathrm{G}$, et al. Isolation of cytotoxic metabolites from targeted peruvian amazonian medicinal plants. J Nat Prod 2008; 71(1): 102-5.

14. Estevez Y, Castillo D, Tangoa PM, Arévalo J, Rojas R, Albán J, Deharo E, Bourdy G, Sauvain M. Evaluation of the leishmanicidal activity of plants used by peruvian chayahuita ethnic group. J Ethnopharmacol 2007; 114: 254-9.

15. Socorro SM, Mendonça FR, Bizzo H, Almeida RI, Soares R, Souto P, Sales A, Hampshire L. Antileishmanial activity of a linalool-rich essential oil from Croton cajucara. Antimicrob Agents Chemother 2003; 47(6): 1895-901.

16. Sánchez LM, Fraga I, Macebo B, Miranda RL. Toxicidad aguda y subaguda oral del extracto acuoso liofilizado de Rhizophora mangle L. en ratas. Rev. Cubana Plant Med 2008; 13(3): 1-13.

Manuscrito recibido el: 03/10/2011

Aceptado para su publicación el: 13/12/2011

\section{Correspondencia:}

Nombre: Luis Inostroza Ruiz

Dirección: Jr. Puno 1002- Lima o1-Perú

e-mail:_linostrozar@unmsm.edu.pe 